\title{
Orchidaceae do Parque Estadual de Ibitipoca, MG, Brasil ${ }^{1}$
}

\author{
Luiz Menini Neto ${ }^{2,6}$, Ruy José Válka Alves³, Fábio de Barros $^{4}$ e Rafaela Campostrini Forzza ${ }^{5}$
}

Recebido em 10/04/2006. Aceito em 17/01/2007

\begin{abstract}
RESUMO - (Orchidaceae do Parque Estadual de Ibitipoca, MG, Brasil). O Parque Estadual de Ibitipoca (PEIB) está situado no sudeste

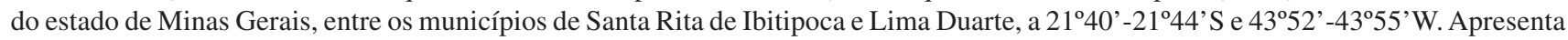
em sua área um mosaico de formações vegetais, das quais o campo rupestre ocupa a maior extensão, sendo também encontradas em seus domínios diversas formações florestais. O presente trabalho teve como objetivo o levantamento das espécies de Orchidaceae ocorrentes no PEIB. Foram registrados 118 táxons distribuídos em 47 gêneros. Os gêneros mais numerosos são Pleurothallis sensu lato (13 spp.), Oncidium (12 spp. e um possível híbrido), Epidendrum (10 spp.) e Maxillaria ( 9 spp.). O estudo da distribuição geográfica dos táxons revelou quatro novos registros para a flora de Minas Gerais e ampliou o conhecimento sobre a distribuição de muitas espécies. Uma comparação com as espécies de orquídeas ocorrentes em outras áreas de campo rupestre conhecidas até o momento demonstra que o PEIB, embora com área relativamente pequena, é uma das regiões com maior número de espécies.
\end{abstract}

Palavras-chave: campo rupestre, Floresta Ombrófila Densa, floresta nebular, biogeografia

ABSTRACT - (Orchidaceae of Ibitipoca State Park, Minas Gerais State, Brazil). Ibitipoca State Park (PEIB) is located in southeastern

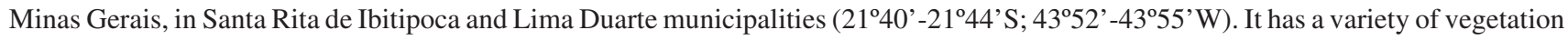
types, with a predominance of "campo rupestre" (rocky grasslands) mixed with forests. This work aimed to survey Orchidaceae species growing at PEIB. One-hundred-and-eighteen taxa belonging to 47 genera were found. The largest genera are Pleurothallis sensu lato (13 spp.), Oncidium (12 spp. and one probable hybrid), Epidendrum (10 spp.), and Maxillaria (9 spp.). Four species are new records for the flora of Minas Gerais state. Compared to other areas of "campos rupestres", PEIB is one of the most species-rich sites, in spite of having a relatively small area.

Key words: "campo rupestre", Atlantic rain forest, cloud forest, biogeography

\section{Introdução}

O Parque Estadual de Ibitipoca (PEIB) está situado no sudeste do Estado de Minas Gerais, entre os municípios de Santa Rita de Ibitipoca e Lima Duarte. Apresenta em sua área um mosaico de formações vegetais, das quais o campo rupestre ocupa a maior extensão. É a menor unidade de conservação do estado que tem o campo rupestre como sua principal formação, sendo também uma das unidades de conservação mais visitadas no Brasil (Salimena-Pires 1997; Rodela 1998; Vitta 2002). Na segunda edição do Atlas para a conservação da biodiversidade no estado de Minas Gerais, a Serra de Ibitipoca figura entre as áreas prioritárias para a conservação da flora no Estado, citada na categoria de importância biológica especial, o nível mais alto adotado (Drummond et al. 2005).

Desde o século XIX há relatos de vários naturalistas que percorreram a Serra de Ibitipoca, como Auguste de Saint-Hilaire (Saint-Hilaire 1822), Carl August Wilhelm Schwacke, em 1896 (Urban 1906) e Álvaro Astolfo da Silveira, no ano de 1912 (Silveira 1928). Geraldo Mendes Magalhães coletou, na Serra de Ibitipoca, material que viria a ser parte de uma listagem preparada por Ferreira \& Magalhães (1977), a primeira realizada para a área, contendo 48 espécies distribuídas em 15 famílias. No fim da década de 1960,

\footnotetext{
1 Parte da Dissertação de Mestrado do primeiro Autor. Programa de pós-graduação em Ciências Biológicas (Botânica) do Museu Nacional/ UFRJ

2 Universidade Federal de Juiz de Fora, Departamento de Botânica, ICB, Bairro Martelos, 36036-330 Juiz de Fora, MG, Brasil

3 Museu Nacional, Departamento de Botânica, 20940-040 Rio de Janeiro, RJ, Brasil

4 Instituto de Botânica, C. Postal 3005, 01061-970 São Paulo, SP, Brasil

5 Jardim Botânico do Rio de Janeiro, Rua Pacheco Leão 915, 22460-030 Rio de Janeiro, RJ, Brasil

6 Autor para correspondência: menini_neto@hotmail.com
} 
o Padre Leopoldo Krieger, então professor da Universidade Federal de Juiz de Fora (UFJF), iniciou seus estudos sobre a flora de Ibitipoca, coletando ao longo das décadas seguintes e formando a coleção base para a Flora da Serra de Ibitipoca no herbário CESJ (Salimena-Pires 1997). Desde então, alguns trabalhos que enfocam a Flora do PEIB foram realizados, apresentando listas de espécies ou tratamentos taxonômicos para algumas famílias (Forzza et al. 1994; Andrade \& Sousa 1995; M.A. Fontes, dados não publicados; Rodela 1998; Carvalho et al. 2000; Menini Neto \& Forzza 2002).

A família Orchidaceae possui aproximadamente 20.000 espécies distribuídas por todo o planeta (Dressler 1993), apresentando alta diversidade no Neotrópico (Christenson 2004). O Brasil abriga cerca de 2.400 espécies (Barros 1996), distribuídas pelos vários ecossistemas, não sendo diferente nos campos rupestres, de modo que muitas vezes figura entre as famílias de maior diversidade (Harley \& Simmons 1986; Giulietti et al. 1987; Peron 1989; Alves 1991; Pirani et al. 1994; 2003; Stannard 1995; Zappi et al. 2003). Os campos rupestres e florestas nebulares são reconhecidos por sua grande riqueza de espécies e alto número de endemismos (Gentry 1992; Giulietti et al.2000), demonstrando a importância dos estudos florísticos no PEIB, em virtude da grande área ocupada por estes dois tipos vegetacionais.

O conhecimento da biodiversidade dos ecossistemas, através de levantamentos florísticos, constitui importante embasamento para a conservação, bem como para uma potencial exploração racional dos recursos e das áreas naturais ainda existentes. Com esse enfoque, o objetivo deste trabalho foi inventariar as espécies de Orchidaceae ocorrentes no Parque Estadual de Ibitipoca, de maneira a ampliar o conhecimento florístico para auxiliar na realização do plano de manejo do Parque. Da mesma forma, o presente estudo é uma contribuição para o conhecimento da Flora de Minas Gerais e da distribuição geográfica das espécies lá ocorrentes.

\section{Material e métodos}

Área de estudo - O PEIB está situado no sudeste do Estado de Minas Gerais, entre os municípios de Santa Rita de Ibitipoca e Lima Duarte, a $21^{\circ} 40^{\prime}-21^{\circ} 44^{\prime} \mathrm{S}$ e $43^{\circ} 52^{\prime}-43^{\circ} 55^{\prime} \mathrm{W}$ (Fig. 1). Inicialmente abrangia uma área de 1.488 ha. da Serra de Ibitipoca, ampliada para $1.923,5$ ha. no ano de 2004, em novas medições nas quais foram inseridos os paredões de seu entorno

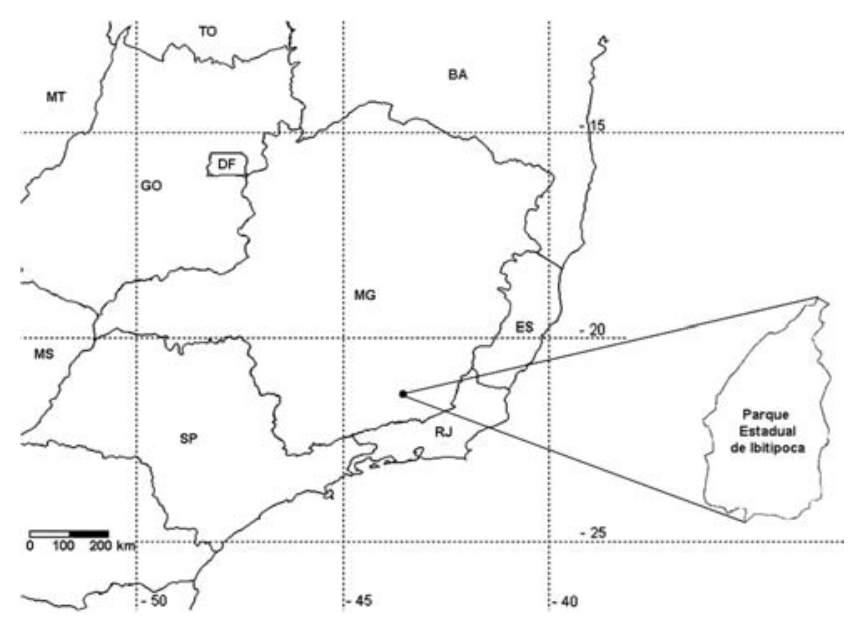

Figura 1. Localização do Parque Estadual de Ibitipoca, MG, Brasil.

(Fig. 4). A área do PEIB é protegida pelo estado desde 1965, sob responsabilidade do Instituto Estadual de Florestas (IEF), tendo-se tornado Parque Estadual em quatro de julho de 1973, pela lei ${ }^{\circ} 6126$ daquele ano (Instituto Estadual de Florestas \& Brandt Meio Ambiente 1994).

A Serra de Ibitipoca faz parte do Complexo da Mantiqueira, cujo relevo caracteriza-se por escarpas altas ou colinas com altitudes variáveis entre $1.200 \mathrm{e}$ 1.800 m (CETEC 1983). A área do PEIB apresenta altitudes destacadas de seu entorno, no qual predominam colinas mais baixas (Rodela 1998). Possui cotas altimétricas médias de 1.500 a $1.600 \mathrm{~m}$, sendo o ponto mais baixo em torno de $1.200 \mathrm{~m}$ de altitude e o ponto culminante, a Lombada ou Pico do Ibitipoca (Fig. 3), na vertente oeste atinge $1.784 \mathrm{~m}$ de altitude. $\mathrm{Na}$ vertente leste, localiza-se o segundo ponto mais alto do Parque, o Pico do Pião (Fig. 2), com $1.721 \mathrm{~m}$ de altitude (Corrêa Neto 1997; Salimena-Pires 1997; Rodela 1998).

O relevo do Parque é bastante escarpado, com paredões e grutas por toda a área. Destacam-se, no relevo, duas cuestas, uma a leste (onde se encontra o Pico do Pião) e outra a oeste (onde se encontra a Lombada), inclinadas para o interior do vale, onde correm o rio do Salto e o córrego da Mata (Corrêa Neto 1997), que se aprofunda para o sul, em direção à queda da cachoeira dos Macacos.

O clima da região é classificado como $\mathrm{Cwb}$ (classificação de Köppen): mesotérmico úmido, com verões amenos e invernos secos. A precipitação anual média é de $1.532 \mathrm{~mm}$ e a temperatura média de $18,9^{\circ} \mathrm{C}$ (CETEC 1983).

As formações vegetacionais do PEIB apresentam-se como um mosaico de vegetações (Fig. 
2-8), havendo várias propostas de denominação de seus tipos. Andrade \& Sousa (1995) dividiram o Parque em quatro formações básicas: campo graminoso, campos rupestres (Fig. 5), campo com arbustos e arvoretas (com predominância da Asteraceae conhecida como candeia - Vanillosmopsis erythropappa Schult. Bip.) e capões de mata. Salimena-Pires (1997) diferenciou seis tipos vegetacionais: campos rupestres (senso estrito), campo rupestre arborizado (Fig. 6), campo gramíneo-lenhoso, mata de galeria, Floresta Estacional Semidecidual Montana e brejo estacional. Rodela (1998) apresentou uma divisão em sete tipos vegetacionais no Parque: remanescentes de Floresta Estacional Semidecidual Montana, Floresta Ombrófila Densa Altimontana, mata ciliar (Fig. 7) e capão de mata, cerrado de altitude, campo rupestre, campo herbáceo-graminoso e campo encharcável. As florestas do interior do PEIB foram estudadas por M.A. Fontes (dados não publicados), demonstrando que devem ser classificadas como Florestas Ombrófilas Densas ou nebulares; esse autor reconheceu duas fisionomias distintas: 1) mata alta, onde se destacam duas áreas denominadas "Mata Grande" e "Matinha", com cerca de 94 e 30 ha, respectivamente, perfazendo mais de $30 \%$ da cobertura total de florestas do PEIB, com árvores de até $25 \mathrm{~m}$, e 2) mata baixa, onde há predominância de "candeia", com árvores alcançando até $12 \mathrm{~m}$.

Confecção da lista de espécies - Foram realizadas coletas bimestrais durante dois anos, entre os meses de outubro/2003 e outubro/2005. As áreas do Parque foram percorridas de forma aleatória, buscando-se cobrir a maior extensão possível em cada expedição. Os espécimes coletados foram incorporados ao herbário CESJ, com duplicatas nos herbários R e RB. As espécies foram identificadas através de consultas às obras de Rodrigues $(1877 ; 1882)$, Cogniaux (1893-1896; 1898-1902; 1904-1906), Hoehne (1940; 1942; 1945; 1949; 1953), Pabst \& Dungs (1975; 1977), Sprunger et al. (1996), além das descrições originais dos táxons e comparação com coleções de vários herbários. Além dos espécimes coletados no período acima citado, a listagem foi elaborada a partir de todos os espécimes coletados no Parque, depositados nos seguintes herbários: BHCB, CESJ, HB, MBM, OUPR, RB, SP, SPF, VIC (acrônimos segundo Holmgren et al. 1990). As informações sobre forma de vida e ambiente foram retiradas das etiquetas dos espécimes ou observadas durante os trabalhos de campo.

\section{Resultados e discussão}

No Parque Estadual do Ibitipoca foram registrados 118 táxons pertencentes a Orchidaceae, distribuídos em 47 gêneros. O gênero que apresenta o maior número de táxons é Pleurothallis sensu lato (13 spp.), seguido por Oncidium (12 spp. e um possível híbrido natural), Epidendrum (10 spp.), Maxillaria (9 spp.), Bulbophyllum (5 spp.), Habenaria (5 spp.), Octomeria (5 spp.) e Stelis (5 spp.). Este número representa um acréscimo considerável em relação às listagens apresentadas por Forzza et al. (1994), Andrade \& Sousa (1995) e Menini Neto \& Forzza (2002) (Tab. 1).

As orquídeas ocorrem no PEIB como epífitas (65 spp. $-\sim 55 \%$ ), rupícolas (36 spp. $-\sim 30 \%$ ) ou terrestres (42 spp. - 35\%), distribuindo-se pelos vários ambientes e formações vegetacionais. Muitas espécies podem apresentar mais de um tipo de preferência por substrato (Tab. 2) mas, de modo geral, as epífitas são encontradas no interior das matas nebulares e ciliares, havendo poucas epífitas nas áreas campestres ou na transição entre as duas formações; as rupícolas ocorrem, em sua maioria, nas áreas campestres do PEIB; já as terrestres apresentam certo equilíbrio numérico entre as áreas de campo e de mata. Deve ser destacado o alto número de espécies epífitas em comparação às terrestres ou rupícolas, fato curioso em se tratando de uma área cuja principal formação vegetacional é o campo rupestre. Isso se dá em conseqüência da presença de áreas relativamente grandes de florestas existentes no interior do Parque, que proporcionam ambiente mais úmido, propício ao desenvolvimento de epífitas (Fig. 8).

A análise de material dos herbários e da literatura revelou algumas espécies raras ou pouco coletadas, tanto na região quanto em outros Estados, como: Lankesterella gnomus, Oncidium divaricatum, O. truncatum, Pleurothallis cryptophoranthoides,

Tabela 1. Comparação entre o número de espécies e gêneros de Orchidaceae citados em listagens realizadas anteriormente para o Parque Estadual de Ibitipoca, MG, Brasil.

\begin{tabular}{lcc}
\hline Listagens & $\begin{array}{c}\text { Número de } \\
\text { espécies }\end{array}$ & $\begin{array}{c}\text { Número de } \\
\text { gêneros }\end{array}$ \\
\hline Forzza et al. (1994) & 69 & 29 \\
Andrade \& Sousa (1995) & 38 & 20 \\
Menini Neto \& Forzza (2002) & 70 & 31 \\
Presente listagem & 118 & 47 \\
\hline
\end{tabular}



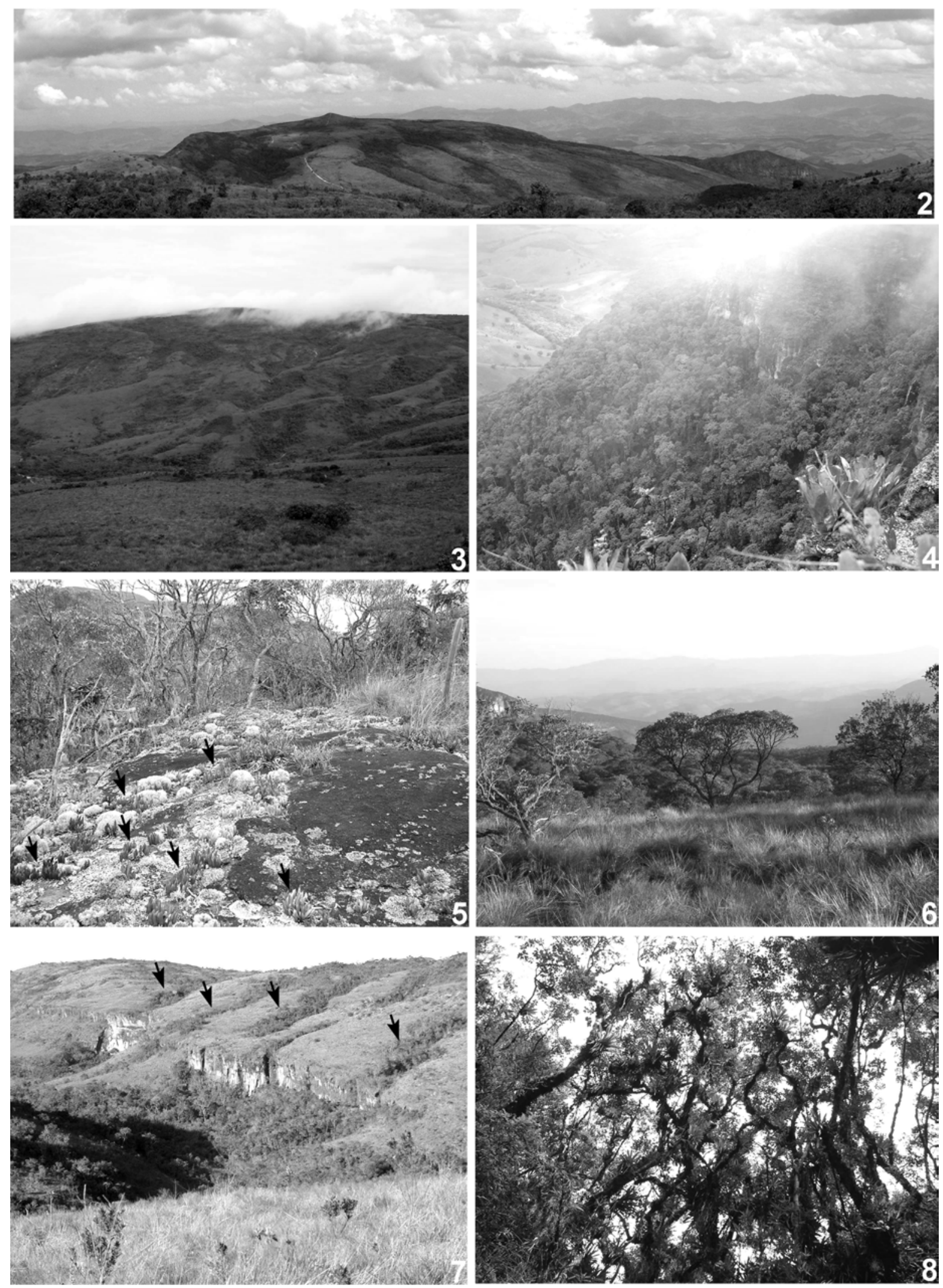

Figuras 2-8. 2. Vista panorâmica da face leste do Parque, com destaque para o Pico do Pião. 3. Vista da face oeste do Parque (Lombada). 4. Mata nebular no paredão da face leste do Parque. 5. Afloramento no campo rupestre (com população de Pleurothallis johannensis, setas). 6. Campo rupestre arbustivo com candeia. 7. Matas ciliares da parte alta do Parque (acima de $1.500 \mathrm{~m}$ alt.) entremeadas ao campo rupestre (setas). 8. Detalhe de epífitas na copa das árvores nas florestas das bordas das grutas (acima de $1.500 \mathrm{~m}$ alt.). 
Tabela 2. Lista das espécies de Orchidaceae registradas no Parque Estadual de Ibitipoca, MG, Brasil.

\begin{tabular}{|c|c|c|c|}
\hline Táxons & Substrato & Habitat & Material testemunho \\
\hline Aspidogyne bidentifera (Schltr.) Garay & $\mathrm{T}$ & $\mathrm{Cr}$ & Sousa s.n. (BHCB 15997) \\
\hline A. commelinoides (Barb. Rodr.) Ames* & $\mathrm{T}$ & Fo & Menini Neto 146 (CESJ) \\
\hline Bifrenaria aureofulva (Hook.) Lindl. & $\mathrm{R}$ & $\mathrm{Cr}$ & Forzza $88(\mathrm{CESJ})$ \\
\hline B. harrisoniae (Hook.) Rchb. f. & $\mathrm{R}$ & $\mathrm{Cr}$ & Forzza 54 (CESJ) \\
\hline B. vitellina Lindl. & $\mathrm{E}$ & Mc & Forzza 8 (CESJ) \\
\hline Bulbophyllum cribbianum Toscano & $\mathrm{E} / \mathrm{R}$ & $\mathrm{Cr}$ & Forzza $10(\mathrm{CESJ})$ \\
\hline B. glutinosum (Barb. Rodr.) Cogn.* & $\mathrm{E}$ & $\mathrm{F}$ & Menini Neto 125 (CESJ) \\
\hline B. luederwaldtii Hoehne \& Schltr.*+ & $\mathrm{E}$ & Mc & Menini Neto 107 (CESJ) \\
\hline B. aff. regnellii Rchb. f.* & $\mathrm{E}$ & $\mathrm{F}$ & Menini Neto 124 (CESJ) \\
\hline B. warmingianum Cogn. & $\mathrm{R}$ & $\mathrm{Cr}$ & Forzza $5(\mathrm{CESJ})$ \\
\hline Cattleya bicolor Lindl.* & $\mathrm{E}$ & $\mathrm{F}$ & Menini Neto 178 (CESJ) \\
\hline C. loddigesii Lindl. & $\mathrm{E}$ & $\mathrm{F}$ & s/coletor (CESJ 27534) \\
\hline Campylocentrum cf. neglectum (Rchb. f. \& Warm.) Cogn.* & $\mathrm{E}$ & Mc & Menini Neto 28 (CESJ) \\
\hline C. cf. robustum Cogn.* & $\mathrm{E}$ & $\mathrm{F}$ & Menini Neto 193 (CESJ) \\
\hline Centroglossa macroceras Barb. Rodr. & $\mathrm{E}$ & Fo & Forzza $54(\mathrm{CESJ})$ \\
\hline Cleistes gracilis Schltr.* & $\mathrm{T}$ & $\mathrm{Cr}$ & Menini Neto 137 (CESJ) \\
\hline C. moritzii (Rchb. f.) Garay \& Dunst. & $\mathrm{T}$ & $\mathrm{Cr}$ & Forzza $22(\mathrm{CESJ})$ \\
\hline Cranichis candida (Barb. Rodr.) Cogn. & $\mathrm{T}$ & Mc & Forzza 43 (CESJ) \\
\hline Cyclopogon lineatus (Lindl.) Pabst & $\mathrm{T}$ & $\mathrm{F}$ & Krieger s.n. (CESJ 9348) \\
\hline Dichaea cogniauxiana Schltr. & $\mathrm{E}$ & $\mathrm{Mc}$ & Forzza $21(\mathrm{CESJ})$ \\
\hline Elleanthus brasiliensis Rchb. f.* & $\mathrm{T} / \mathrm{R}$ & $\mathrm{F}$ & Menini Neto 126 (CESJ) \\
\hline Encyclia patens Hook.* & $\mathrm{E}$ & $\mathrm{F}$ & Menini Neto 165 (CESJ) \\
\hline Epidendrum armeniacum Lindl.* & $\mathrm{E}$ & $\mathrm{F}$ & Menini Neto 175 (CESJ) \\
\hline E. chlorinum Barb. Rodr.* & $\mathrm{E}$ & Mc & Menini Neto 171 (CESJ) \\
\hline E. dendrobioides Thunb. & $\mathrm{T} / \mathrm{R}$ & $\mathrm{Cr}$ & Forzza $68(\mathrm{CESJ})$ \\
\hline E. difforme Lindl.* & $\mathrm{E}$ & $\mathrm{F}$ & Menini Neto 97 (CESJ) \\
\hline E. martianum Lindl. & $\mathrm{T} / \mathrm{R}$ & $\mathrm{Cr}$ & Forzza $72(\mathrm{CESJ})$ \\
\hline E. ochrochlorum Barb. Rodr.+ & $\mathrm{E}$ & Fo & Forzza 36 (CESJ) \\
\hline E. paranaense Barb. Rodr. & $\mathrm{E}$ & $\mathrm{Fo} / \mathrm{Mc}$ & Forzza 83 (CESJ) \\
\hline E. ramosum Jacq. & $\mathrm{R}$ & $\mathrm{Cr}$ & Forzza 16 (CESJ) \\
\hline E. rigidum Jacq.* & $\mathrm{E}$ & $\mathrm{Mc}$ & Menini Neto 71 (CESJ) \\
\hline E. secundum Jacq. & $\mathrm{T} / \mathrm{R}$ & $\mathrm{Cr}$ & Forzza $7(\mathrm{CESJ})$ \\
\hline Eryhtrodes austrobrasiliensis (Porsch) Pabst*+ & $\mathrm{T}$ & $\mathrm{Cr}$ & Menini Neto 147 (CESJ) \\
\hline Eurystyles cogniauxii (Kraenzl.) Pabst & $\mathrm{E}$ & Mc & Forzza $61(\mathrm{CESJ})$ \\
\hline Galeandra beyrichii Rchb. f.* & $\mathrm{T}$ & Fo & Menini Neto 117 (CESJ) \\
\hline Gomesa glaziovii Cogn. & $\mathrm{T} / \mathrm{E}$ & $\mathrm{Mc}$ & Forzza $51(\mathrm{CESJ})$ \\
\hline G. recurva Lodd. & $\mathrm{E}$ & Fo & Forzza 38 (CESJ) \\
\hline Grobya amherstiae Lindl. & $\mathrm{E}$ & $\mathrm{F}$ & Forzza 26 (CESJ) \\
\hline Habenaria josephensis Barb. Rodr.* & $\mathrm{T}$ & $\mathrm{Cr}$ & Menini Neto 153 \\
\hline H. aff. mitomorpha Kraenzl. & $\mathrm{T}$ & $\mathrm{Cr}$ & s/coletor (CESJ 29039) \\
\hline H. parviflora Lindl.* & $\mathrm{T}$ & $\mathrm{Cr}$ & Menini Neto 151 (CESJ) \\
\hline H. rolfeana Schltr. & $\mathrm{T}$ & $\mathrm{Cr}$ & Forzza $82(\mathrm{CESJ})$ \\
\hline H. rupicola Barb. Rodr. & $\mathrm{T}$ & $\mathrm{Cr}$ & Krieger s.n. (CESJ 8575) \\
\hline Hadrolaelia coccinea (Lind1.) Chiron \& V. P. Castro & $\mathrm{E} / \mathrm{R}$ & $\mathrm{F} / \mathrm{Cr}$ & Forzza $17(\mathrm{CESJ})$ \\
\hline Hoffmannseggella caulescens (Lind1.) Chiron \& V. P. Castro & $\mathrm{R}$ & $\mathrm{Cr}$ & Sousa s.n. (BHCB 16751) \\
\hline H. crispata (Thunb.) H.G. Jones & $\mathrm{R}$ & $\mathrm{Cr}$ & Forzza $18(\mathrm{CESJ})$ \\
\hline Isabelia violacea (Lind1.) Van den Berg \& M.W. Chase & $\mathrm{E} / \mathrm{R}$ & $\mathrm{Cr}$ & Salimena-Pires s.n. (CESJ 25458) \\
\hline I. virginalis Barb. Rodr.* & $\mathrm{E}$ & $\mathrm{F}$ & Menini Neto 47 (CESJ) \\
\hline Isochilus linearis (Jacq.) R. Br. & $\mathrm{E}$ & $\mathrm{F}$ & Forzza 40 (CESJ) \\
\hline Lankesterella gnomus (Kraenzl.) Hoehne* & $\mathrm{E}$ & Mc & Menini Neto 139 (CESJ) \\
\hline Malaxis excavata Kuntze & $\mathrm{T}$ & $\mathrm{Mc}$ & Menini Neto 72 (CESJ) \\
\hline Masdevallia infracta Lindl.* & $\mathrm{E}$ & Fo & Menini Neto 173 (CESJ) \\
\hline Maxillaria acicularis Herb. ex Lindl. & $\mathrm{E}$ & $\mathrm{F}$ & Forzza 35 (CESJ) \\
\hline M. brasiliensis Brieger \& Illg & $\mathrm{R}$ & $\mathrm{F}$ & Forzza 3 (CESJ) \\
\hline M. aff. crysantha Barb. Rodr.* & $\mathrm{R}$ & $\mathrm{F}$ & Menini Neto 167 (CESJ) \\
\hline M. gracilis Lindl.* & $\mathrm{T} / \mathrm{R}$ & $\mathrm{Cr} / \mathrm{Fo}$ & Menini Neto 170 (CESJ) \\
\hline
\end{tabular}


Tabela 2 (continuação)

\begin{tabular}{|c|c|c|c|}
\hline Táxons & Substrato & Habitat & Material testemunho \\
\hline M. madida Lindl. & $\mathrm{E} / \mathrm{R}$ & $\mathrm{Cr}$ & Forzza $47(\mathrm{CESJ})$ \\
\hline M. notylioglossa Rchb. f.* & $\mathrm{E}$ & $\mathrm{F}$ & Menini Neto 119 (CESJ) \\
\hline M. ochroleuca Lodd. ex Lindl. & $\mathrm{E}$ & $\mathrm{F}$ & Forzza $71(\mathrm{CESJ})$ \\
\hline M. rigida Barb. Rodr.* & $\mathrm{R}$ & $\mathrm{Cr}$ & Menini Neto 29 (CESJ) \\
\hline M. rupestris Lindl. & $\mathrm{R}$ & $\mathrm{Cr}$ & Forzza $92(\mathrm{CESJ})$ \\
\hline Mesadenella sp.* & $\mathrm{T}$ & $\mathrm{Mc}$ & Menini Neto 179 (CESJ) \\
\hline Octomeria alpina Barb. Rodr. & $\mathrm{E} / \mathrm{R}$ & $\mathrm{Cr} / \mathrm{Mc}$ & Forzza $77(\mathrm{CESJ})$ \\
\hline$O$. aff. diaphana Lindl. & $\mathrm{E}$ & Fo & Forzza 89 (CESJ) \\
\hline O. grandiflora Lindl. & $\mathrm{E}$ & $\mathrm{Mc}$ & Forzza 63 (CESJ) \\
\hline O. aff. rubrifolia Barb. Rodr. & $\mathrm{E}$ & $\mathrm{F} / \mathrm{Cr}$ & Sousa s.n. (BHCB 14766) \\
\hline O. wawrae Rchb. f. ex Wawra & $\mathrm{E}$ & $\mathrm{F} / \mathrm{Cr}$ & Forzza $41(\mathrm{CESJ})$ \\
\hline Oncidium aff. batemannianum Parm. ex Knowles \& Westc. & $\mathrm{T} / \mathrm{R}$ & $\mathrm{Cr}$ & Menini Neto 81 (CESJ) \\
\hline O. divaricatum Lindl.* & $\mathrm{E}$ & Fo & Forzza $2190(\mathrm{RB})$ \\
\hline O. donianum Bateman ex W. Baxter & $\mathrm{T} / \mathrm{R}$ & $\mathrm{Cr}$ & Forzza 50 (CESJ) \\
\hline O. gravesianum Rolfe & $\mathrm{E}$ & Fo & Forzza 23 (CESJ) \\
\hline O. hookeri Rolfe & $\mathrm{E}$ & $\mathrm{F} / \mathrm{Fo}$ & Forzza $39(\mathrm{CESJ})$ \\
\hline O. longipes Lindl.* & $\mathrm{E}$ & $\mathrm{F}$ & Menini Neto 163 (CESJ) \\
\hline O. pirarense Rchb. f. & $\mathrm{T} / \mathrm{R}$ & $\mathrm{Cr}$ & Forzza $80(\mathrm{CESJ})$ \\
\hline O. truncatum Pabst* & $\mathrm{E}$ & $\mathrm{F}$ & Menini Neto 95 (CESJ) \\
\hline O. varicosum Lindl.* & $\mathrm{R}$ & $\mathrm{Cr}$ & Menini Neto 144 (CESJ) \\
\hline O. warmingii Rchb. f. & $\mathrm{T} / \mathrm{R}$ & $\mathrm{Cr}$ & Forzza $6(\mathrm{CESJ})$ \\
\hline Oncidium sp. $1 *$ & $\mathrm{~T} / \mathrm{R}$ & $\mathrm{Cr}$ & Menini Neto 128 (CESJ) \\
\hline Oncidium sp. $2^{*}$ & $\mathrm{~T} / \mathrm{R}$ & $\mathrm{Cr}$ & Menini Neto 109 (CESJ) \\
\hline Oncidium cf. híbrido $(O$. batemannianum $\times$ O. warmingii) & $\mathrm{T} / \mathrm{R}$ & $\mathrm{Cr}$ & Menini Neto 73 (CESJ) \\
\hline Pleurothallis cryptophoranthoides Loefgr. & $\mathrm{E}$ & Fo & Sousa s.n. (BHCB 16647) \\
\hline P. heterophylla (Barb. Rodr.) Cogn. & $\mathrm{E}$ & $\mathrm{F}$ & Andrade 1160 (BHCB) \\
\hline P. hypnicola Lindl.* & $\mathrm{E}$ & Fo & Menini Neto 134 (CESJ) \\
\hline P. johannensis Barb. Rodr. & $\mathrm{R}$ & $\mathrm{Cr}$ & Forzza $11(\mathrm{CESJ})$ \\
\hline P. luteola Lindl.* & $\mathrm{E}$ & Fo & Menini Neto 158 (CESJ) \\
\hline P. malachantha Rchb. f. & $\mathrm{E}$ & $\mathrm{F} / \mathrm{Fo}$ & Sousa s.n. (BHCB 9832) \\
\hline P. marginalis Rchb. f.* & $\mathrm{E}$ & F & Menini Neto 162 (CESJ) \\
\hline P. modestissima $\mathrm{Rchb} . \mathrm{f}$. & $\mathrm{T} / \mathrm{R}$ & $\mathrm{F} / \mathrm{Cr}$ & Forzza $19(\mathrm{CESJ})$ \\
\hline P. quartzicola (Barb. Rodr.) Cogn.* & $\mathrm{E}$ & Fo & Menini Neto 114 (CESJ) \\
\hline$P$. recurva Lindl.* & $\mathrm{E}$ & Fo & Menini Neto 236 (CESJ) \\
\hline P. rubens Lindl. & $\mathrm{E} / \mathrm{R}$ & $\mathrm{Cr} / \mathrm{F} / \mathrm{Fo}$ & Forzza $78(\mathrm{CESJ})$ \\
\hline P. saundersiana $\mathrm{Rchb} . \mathrm{f}$. & $\mathrm{E}$ & $\mathrm{F}$ & Forzza $32(\mathrm{CESJ})$ \\
\hline P. tricarinata Poepp. \& Endl.* & $\mathrm{E}$ & Fo & Menini Neto 118 (CESJ) \\
\hline Pogoniopsis schenckii Cogn. & $\mathrm{T}$ & Fo & Sousa s.n. (BHCB 14671) \\
\hline Polystachya hoehneana Kraenzl. & $\mathrm{E}$ & $\mathrm{F}$ & Forzza $37(\mathrm{CESJ})$ \\
\hline Ponthieva pubescens (C. Presl.) C. Schweinf. & $\mathrm{T}$ & $\mathrm{F}$ & Krieger s.n. (CESJ 14707) \\
\hline Prescottia montana Barb. Rodr. & $\mathrm{T}$ & $\mathrm{Cr}$ & Forzza $44(\mathrm{CESJ})$ \\
\hline P. stachyodes Lindl.* & $\mathrm{T}$ & $\mathrm{F}$ & Menini Neto 33 (CESJ) \\
\hline Promenaea xanthina Lindl. & $\mathrm{E}$ & Fo & Eiterer s.n. (CESJ 25549) \\
\hline Prosthechea allemanoides (Hoehne) W.E. Higgins & $\mathrm{E} / \mathrm{R}$ & $\mathrm{F} / \mathrm{Cr}$ & Forzza $55(\mathrm{CESJ})$ \\
\hline P. vespa (Vell.) W.E. Higgins & $\mathrm{E} / \mathrm{R}$ & $\mathrm{F} / \mathrm{Cr}$ & Forzza $67(\mathrm{CESJ})$ \\
\hline Prosthechea sp.* & $\mathrm{R}$ & $\mathrm{Mc}$ & Menini Neto 180 (CESJ) \\
\hline Psilochilus sp.* & $\mathrm{T}$ & Fo & Menini Neto 181 (CESJ) \\
\hline Rodrigueziella gomezoides (Barb. Rodr.) Berman & $\mathrm{E}$ & $\mathrm{Mc}$ & Sousa s.n. (BHCB 16117) \\
\hline Sacoila lanceolata (Aubl.) Garay & $\mathrm{T}$ & $\mathrm{Cr}$ & Sousa s.n. (BHCB 15772) \\
\hline Sauroglossum nitidum (Vell.) Schltr.* & $\mathrm{T}$ & $\mathrm{F}$ & Menini Neto 182 (CESJ) \\
\hline Scaphyglottis modesta Schltr. & $\mathrm{T} / \mathrm{E}$ & $\mathrm{F}$ & Forzza $4(\mathrm{CESJ})$ \\
\hline Scuticaria aff. kautskyi Pabst & $\mathrm{E}$ & $\mathrm{Mc}$ & Forzza $15(\mathrm{CESJ})$ \\
\hline Stelis aprica Lindl.* & $\mathrm{E}$ & $\mathrm{Mc}$ & Menini Neto 127 (CESJ) \\
\hline S. intermedia Poepp. \& Endl.* & $\mathrm{E}$ & Fo & Menini Neto 159 (CESJ) \\
\hline S. megantha Barb. Rodr. & $\mathrm{E}$ & Fo & Forzza $60(\mathrm{CESJ})$ \\
\hline S. papaquerensis Rchb. f. & $\mathrm{E}$ & Fo & Sousa s.n. (BHCB 14764) \\
\hline S. parvula Lindl.+ & $\mathrm{E}$ & $\mathrm{Mc}$ & Krieger s.n. (CESJ 8593) \\
\hline
\end{tabular}


Tabela 2 (continuação)

\begin{tabular}{lccc}
\hline Táxons & Substrato & Habitat & Material testemunho \\
\hline Stigmatosema polyaden (Vell.) Garay & $\mathrm{T}$ & Fo & Forzza 56 (CESJ) \\
Thysanoglossa organensis Brade* & $\mathrm{E}$ & $\mathrm{F}$ & Menini Neto 89 (CESJ) \\
Zygopetalum cf. brachypetalum Lindl. & $\mathrm{T} / \mathrm{R}$ & $\mathrm{Cr}$ & Forzza 24 (CESJ) \\
Z. mackaii Hook. & $\mathrm{T} / \mathrm{R}$ & $\mathrm{Cr}$ & Forzza 12 (CESJ) \\
Z. triste Barb. Rodr.* & $\mathrm{T} / \mathrm{R}$ & $\mathrm{Cr}$ & Menini Neto 70 (CESJ) \\
\hline
\end{tabular}

Cada exemplar citado corresponde à primeira coleta do material no PEIB. Preferência por substrato: $\mathrm{E}=$ epífita, $\mathrm{T}=$ terrestre, $\mathrm{R}=$ rupícola . Habitat: $\mathrm{Fo}=$ Floresta ombrófila; $\mathrm{Cr}=$ Campo rupestre; $\mathrm{Mc}=$ Mata ciliar ou matas próximas às grutas (matas da parte alta do parque, acima de $1.500 \mathrm{~m}$ alt.); F = Floresta Estacional Semidecidual (capões de mata da parte baixa do Parque, $1.200 \mathrm{~m}$ alt.). *Acréscimo às listas de espécies publicadas para o PEIB (Ferreira \& Magalhães 1977; Forzza et al. 1994; Andrade \& Sousa 1995; Menini Neto \& Forzza 2002). Os novos registros para a flora de Minas Gerais estão destacados com o símbolo +.

P. malachantha, P. quartzicola, Pogoniopsis schenckii, Polystachya hoehneana, Ponthieva pubescens e Stelis parvula. É possível que estas espécies sejam realmente raras ou estejam ameaçadas em virtude da destruição de seus habitats. Por outro lado, a lacuna de conhecimento e registro destas espécies pode ser devida ao esforço de coleta insuficiente. Desse modo, mais estudos são necessários para se determinar a real situação de conservação destes táxons.

Novas ocorrências para Minas Gerais, através de material coletado no PEIB foram citadas na listagem preparada por Andrade \& Sousa (1995), embora não tenham sido destacadas por aqueles autores no referido trabalho. São elas: Pleurothallis cryptophoranthoides, $P$. heterophylla e P. malachantha. Deve-se destacar a ocorrência de Stelis parvula, uma espécie anteriormente citada para o México e América Central (http://www.kew.org/ monocotChecklist), (World Checklist of Monocots 2004) e para o Pico das Almas, Bahia (Toscano-de-Brito 1995). A espécie é aqui registrada para Minas Gerais e Rio de Janeiro.

Uma comparação do número de espécies de Orchidaceae encontradas no PEIB com o de outras áreas já inventariadas, demonstra que no PEIB há uma grande concentração de espécies em uma área relativamente pequena, compatível com outras áreas da Floresta Atlântica, região reconhecidamente rica em espécies de orquídeas (Tab. 3).

Alguns padrões de distribuição das espécies registradas no PEIB puderam ser verificados com base na literatura (Pabst \& Dungs 1975; 1977; Sprunger et al. 1996; World Checklist of Monocots 2004; Toscano de Brito \& Cribb 2005) e no material examinado nos herbários visitados:

1) espécies de ampla distribuição ao longo da região neotropical: Cyclopogon lineatus, Epidendrum difforme, E. ramosum, E. rigidum, E. secundum, Galeandra beyrichii, Isochilus linearis, Malaxis excavata, Octomeria grandiflora, Prosthechea vespa, Prescottia stachyodes, Sacoila lanceolata, Scaphyglottis modesta e Stelis aprica;

2) espécies com distribuição ampla, mas descontínua, na América do Sul: Elleanthus brasiliensis, Epidendrum armeniacum, E. dendrobioides, Habenaria parviflora, Maxillaria notylioglossa, $M$. ochroleuca, Stelis intermedia e S. papaquerensis;

3) espécies distribuídas predominantemente no domínio da Floresta Atlântica e nos Andes: Epidendrum armeniacum, Masdevallia infracta, Pleurothallis rubens, $P$. saundersiana, $P$. tricarinata e Ponthieva pubescens;

4) espécies distribuídas pelos estados das regiões Sudeste e Sul do Brasil e Argentina: Aspidogyne bidentifera, Cattleya loddigesii, Cranichis candida, Gomesa recurva, Isabelia virginalis, Maxillaria rupestris, Oncidium longipes, O. varicosum e Stigmatosema polyaden;

5) espécies distribuídas pelos estados das regiões Sudeste e Sul e Bahia: Bifrenaria aureofulva, Dichaea cogniauxiana, Grobya amherstiae, Maxillaria gracilis, Oncidium hookeri, Pleurothallis heterophylla e Zygopetalum mackayi;

6) espécies com distribuição predominante no domínio da Floresta Atlântica nas regiões Sudeste e Sul do Brasil: Aspidogyne commelinoides, Bifrenaria harrisoniae, B. vitellina, Bulbophyllum glutinosum, B. luederwaldtii, Cattleya loddigesii, Centroglossa macroceras, Epidendrum parahybunense, Eurystyles cogniauxii, Gomesa glaziovii, Habenaria rolfeana, Maxillaria acicularis, M. brasiliensis, M. madida, Octomeria alpina, O. diaphana, O. wawrae, Oncidium truncatum, Pleurothallis cryptophoranthoides, P. hypnicola, P. luteola, 
Tabela 3. Comparação entre o número de gêneros e espécies de Orchidaceae registradas em levantamentos diversos no Brasil. P.N. = Parque Nacional; P.E. = Parque Estadual; R.B. = Reserva Biológica; R.F. = Reserva Florestal.

\begin{tabular}{|c|c|c|c|}
\hline Local (Estado) & $\mathrm{N}^{\circ}$ gêneros/táxons & Área & Referência \\
\hline \multicolumn{4}{|l|}{ Cerrado/campo rupestre } \\
\hline Mucugê (BA) & $13 / 26$ & 89.600 ha & Harley \& Simmons 1986 \\
\hline P. N. Serra do Cipó (MG) & $33 / 80$ & 33.800 ha & Barros 1987 \\
\hline P. E. Itacolomi (MG) & $24 / 41$ & 7.543 ha & Alves 1990 \\
\hline Serra de São José (MG) & $40 / 86$ & 2.500 ha & Alves 1991 \\
\hline Pico das Almas (BA) & $19 / 46$ & $17.000 \mathrm{ha}$ & Toscano-de-Brito 1995 \\
\hline Estação Ecológica de Tripuí (MG) & $7 / 10$ & 337 ha & Pedralli et al. 1997 \\
\hline P. N. da Chapada dos Veadeiros (GO) & $18 / 47$ & 65.000 ha & Munhoz \& Proença 1998 \\
\hline Catolés (BA) & $31 / 76$ & 66.700 ha & Zappi et al. 2003 \\
\hline Grão Mogol (MG) & $19 / 34$ & $33.324,72$ & Barros \& Pinheiro 2004 \\
\hline Reserva Ecológica do Guará (DF) & $44 / 105$ & 147 ha & Batista et al. 2005 \\
\hline Chapada Diamantina (BA) & $65 / 175$ & ? & Toscano-de-Brito \& Cribb 2005 \\
\hline \multicolumn{4}{|l|}{ Floresta Atlântica } \\
\hline P. E. das Fontes do Ipiranga (SP) & $52 / 125$ & 345 ha & Barros 1983 \\
\hline P. E. da Ilha do Cardoso (SP) & $53 / 118$ & 22.500 ha & Barros 1991 \\
\hline Macaé de Cima (RJ) & $66 / 270$ & 15.000 ha & Miller et al. 1996 \\
\hline P. N. do Caparaó (MG) & $31 / 86$ & 31.853 ha & Leoni 1997 \\
\hline R. B. da Represa do Grama (MG) & $23 / 28$ & 264 ha & Menini Neto et al. 2004 \\
\hline P. E. da Serra do Brigadeiro (MG) & $48 / 106$ & 13.210 ha & Leoni \& Tinte 2004 \\
\hline P. E. de Ibitipoca (MG) & $47 / 118$ & $1.923,5$ ha & Presente Trabalho \\
\hline \multicolumn{4}{|l|}{ Floresta Amazônica } \\
\hline R. B. de Campina (AM) & $17 / 31$ & 900 ha & Braga 1977 \\
\hline Serra dos Carajás (PA) & $55 / 118$ & 429.000 ha & Silveira et al. 1995 \\
\hline Serra das Andorinhas (PA) & $37 / 76$ & 60.000 ha & Atzingen et al. 1996 \\
\hline R. F. Adolpho Ducke (AM) & $40 / 78$ & $10.072 \mathrm{ha}$ & Ribeiro et al. 1999 \\
\hline
\end{tabular}

P. malachantha, P. marginalis, P. quartzicola, Promenaea xanthina, Prosthechea allemanoides, Polystachya hoehneana, Stelis megantha, Thysanoglossa organensis e Zygopetalum triste; 7) espécies exclusivas dos campos rupestres do estado de Minas Gerais: Hoffmannseggella caulescens, H. crispata, Pleurothallis modestissima (Cadeia do Espinhaço e campos rupestres do sul e sudeste do estado), $P$. johannensis (apenas nos campos do sul e sudeste de $\mathrm{MG}$ ).

Conservação - O Parque Estadual de Ibitipoca é muito visitado por turistas ao longo de todo o ano. Estima-se que o número de visitantes alcance os 40.000 por ano (Menini Neto \& Forzza 2002). Tal visitação muitas vezes coloca em risco populações de espécies com alto potencial ornamental. Além da pressão de coleta ilegal exercida por parte dos turistas, a criação de trilhas sem a orientação dos funcionários, bem como a erosão de trilhas já existentes, pode atingir diretamente as populações de algumas espécies.

Dentre as espécies registradas no Parque, seis figuram na Lista Vermelha das Espécies Ameaçadas de Extinção da Flora de Minas Gerais (Mendonça \&
Lins 2000), sendo apresentadas com status variados: Bulbophyllum warmingianum, Cattleya bicolor, C. loddigesii, Hadrolaelia coccinea (citada como Sophronitis coccinea), Oncidium warmingii e Zygopetalum triste. Destas, apenas B. warmingianum e $O$. warmingii apresentam populações grandes e largamente distribuídas pela área do Parque. As espécies de Cattleya são conhecidas apenas de uma coleta cada, sendo muito raras no local. Hadrolaelia coccinea, segundo informações de funcionários e guias do Parque, é a espécie que mais sofreu com a coleta predatória exercida pelos visitantes, tendo seu número reduzido visivelmente nos últimos anos, em virtude de seu alto potencial ornamental e pequeno tamanho, o que facilita sua retirada do local. Zygopetalum triste ainda apresenta algumas pequenas populações na parte alta do Parque (acima dos $1.500 \mathrm{~m}$ de altitude).

\section{Agradecimentos}

Ao IEF-MG, em especial à administração e aos funcionários do Parque por todo apoio e incentivo para o desenvolvimento deste trabalho; à FAPERJ pela bolsa 
concedida ao primeiro autor (processo E-26/151779/ 2003); ao Programa de Pós-graduação em Ciências Biológicas (Botânica) do Museu Nacional/UFRJ; ao CNPq, pela Bolsa de Produtividade em Pesquisa recebida pelo terceiro autor (processo 303962/2004-6); ao Dr. Jefferson Prado pelo auxílio na revisão do abstract.

\section{Referências bibliográficas}

Alves, R.J.V. 1990. The Orchidaceae of Itacolomi State Park in Minas Gerais, Brazil. Acta Botanica Brasilica 4: 65-72.

Alves, R.J.V. 1991. Guia de campo das orquídeas da Serra de São José, MG, Brasil. Tropicaleaf.

Andrade, P.M. \& Sousa, H.C. 1995. Contribuição ao conhecimento da vegetação do Parque Estadual de Ibitipoca, Lima Duarte, Minas Gerais. Revista Árvore 19: $249-261$.

Atzingen, N.V.; Cardoso, A.L.R. \& Ilkiu-Borges, A.L. 1996. Flora orquidológica da Serra das Andorinhas, São Geraldo do Araguaia - PA. Boletim do Museu Paraense Emílio Goeldi, série Botânica 12: 59-74.

Barros, F. 1983. Flora fanerogâmica da reserva do Parque Estadual das Fontes do Ipiranga (São Paulo, Brasil.). 198 - Orchidaceae. Hoehnea 10: 74-124.

Barros, F. 1987. Orchidaceae. Pp. 125-130. In: A.M. Giulietti; N.L. Menezes; J.R. Pirani; M. Meguro \& M.G.L. Wanderley (eds.). Flora da Serra do Cipó, Minas Gerais: caracterização e lista das espécies. Boletim de Botânica da Universidade de São Paulo 9: 1-151.

Barros, F. 1991. Orchidaceae. Pp. 142-152. In: M.M.R.F. Melo; F. Barros; M.G.L. Wanderley; M. Kirizawa; S.L. JungMendaçolli \& S.A.C. Chiea (eds.). Flora fanerogâmica da Ilha do Cardoso: Caracterização geral da vegetação e listagem das espécies ocorrentes, v. 1. São Paulo, Instituto de Botânica.

Barros, F. 1996. Notas taxonômicas para as espécies brasileiras dos gêneros Epidendrum, Platystele, Pleurothallis e Scaphyglottis (Orchidaceae). Acta Botanica Brasilica 10: 139-151.

Barros, F. \& Pinheiro, F. 2004. Flora de Grão-Mogol, Minas Gerais: Orchidaceae. Boletim de Botânica da Universidade de São Paulo 22: 361-383.

Batista, J.A.N.; Bianchetti, L.B \& Pellizzaro, K.F. 2005. Orchidaceae da Reserva Biológica do Guará, DF, Brasil. Acta Botanica Brasilica 19: 221-232.

Braga, P.I.S. 1977. Aspectos biológicos das Orchidaceae de uma campina da Amazônia Central. Acta Amazonica (suplemento) 7: 1-89.

Carvalho, L.M.T.; Fontes, M.A. \& Oliveira Filho, A. 2000. Tree species distribution in canopy gaps and mature forest in an area of cloud forest of the Ibitipoca Range, south-eastern Brazil. Plant Ecology 149: 9-22.

CETEC. 1983. Diagnóstico ambiental de Minas Gerais. Belo Horizonte.
Christenson, E. 2004. Orchidaceae. Pp. 465-468. In: N. Smith; S. Mori; A. Henderson; D.W. Stevenson \& S.V. Heald (eds.). Flowering Plants of Neotropics. Princeton, Princeton University Press.

Cogniaux, A. 1893-1896. Orchidaceae. Pp. 1-672. In: C.F.P. Martius, A.G. Eichler \& I. Urban (eds.). Flora brasiliensis 3(4). Monachii, Typographia Regia.

Cogniaux, A. 1898-1902. Orchidaceae. Pp. 1-664. In: C.F.P. Martius, A.G. Eichler \& I. Urban (eds.). Flora brasiliensis 3(5). Monachii, Typographia Regia.

Cogniaux, A. 1904-1906. Orchidaceae. Pp. 1-604. In: C.F.P. Martius; A.G. Eichler \& I. Urban (eds.). Flora brasiliensis 3(6). Monachii, Typographia Regia.

Corrêa Neto, A.V. 1997. Cavernas em quartzitos da Serra do Ibitipoca, sudeste de Minas Gerais. Pp. 51-60. In: G.C. Rocha (coord.). Anais do $1^{\circ}$ Seminário de Pesquisa sobre o Parque Estadual de Ibitipoca. Juiz de Fora, Núcleo de Pesquisa em Zoneamento Ambiental da UFJF.

Dressler, R.L. 1993. Phylogeny and Classification of the Orchid Family. Portland, Dioscorides Press.

Drummond, G.M.; Martins, C.S.; Machado, A.B.M; Sebaio, F.A. \& Antonini, Y (orgs.). 2005. Biodiversidade em Minas Gerais, um atlas para sua conservação. $2^{\mathrm{a}}$ ed. Belo Horizonte, Fundação Biodiversitas.

Ferreira, M.B \& Magalhães, G.M. 1977. Contribuição para o conhecimento da vegetação da Serra do Espinhaço em Minas Gerais (Serras de Grão Mogol e da Ibitipoca). Pp. 189-202. In: Anais do XXVI Congresso Nacional de Botânica, 1975. Rio de Janeiro.

Forzza, R.C.; Barros, F. \& Salimena-Pires, F.R. 1994. Orchidaceae do Parque Estadual de Ibitipoca, Minas Gerais (checklist). Principia 1: 125-136.

Gentry, A.H. 1992. Tropical forest biodiversity: distributional patterns and their conservational significance. Oikos 63: $19-28$.

Giulietti, A.M.; Menezes, N.L.; Pirani, J.R.; Meguro, M. \& Wanderley, M.G.L. 1987. Flora da Serra do Cipó, Minas Gerais: Caracterização e lista das espécies. Boletim de Botânica da Universidade de São Paulo 9: 1-151.

Giulietti, A.M.; Harley, R.M.; Queiroz, L.P.; Wanderley, M.G.L. \& Pirani, J.R. 2000. Caracterização e endemismos nos campos rupestres da Cadeia do Espinhaço. Pp. 311-318. In: T.B. Cavalcanti \& B.M.T. Walter (eds.). Tópicos Atuais em Botânica. Brasília, SBB/Embrapa.

Harley, R.M. \& Simmons, N.A. 1986. Florula of Mucugê: Chapada Diamantina - Bahia, Brazil. Kew, Royal Botanic Gardens.

Hoehne, F.C. 1940. Orchidaceas. Pp. 1-254. In: F.C. Hoehne (ed.). Flora Brasilica 12(1). São Paulo, Secretaria da Agricultura, Indústria e Comércio de São Paulo.

Hoehne, F.C. 1942. Orchidaceas. Pp. 1-218. In: F.C. Hoehne (ed.). Flora Brasilica 12(6). São Paulo, Secretaria da Agricultura, Indústria e Comércio de São Paulo.

Hoehne, F.C. 1945. Orchidaceas. Pp. 1-389. In: F.C. Hoehne (ed.). Flora Brasilica 12(2). São Paulo, Secretaria da Agricultura, Indústria e Comércio de São Paulo.

Hoehne, F.C. 1949. Iconografia das Orchidaceas do Brasil. São Paulo, Secretaria da Agricultura. 
Hoehne, F.C. 1953. Orchidaceas. Pp. 1-397. In: F.C. Hoehne (ed.). Flora Brasilica 12(7). São Paulo, Secretaria da Agricultura, Indústria e Comércio de São Paulo.

Holmgren, P.K.; Holmgren, N.H. \& Barnett, L.C. 1990. Index Herbariorum: the herbaria of the world. New York, New York Botanical Garden.

Instituto Estadual de Florestas \& Brandt Meio Ambiente. 1994. Parque Estadual de Ibitipoca: levantamento dos aspectos históricos e culturais. Belo Horizonte.

Leoni, L.S. 1997. Catálogo preliminar das fanerógamas ocorrentes no Parque Nacional do Caparaó, Minas Gerais. Pabstia 8: 1-28.

Leoni, L.S. \& Tinte, V.A. 2004. Flora do Parque Estadual da Serra do Brigadeiro, estado de Minas Gerais, Brasil v. I - Caracterização da vegetação e lista preliminar das espécies. Carangola, Gráfica São José.

Menini Neto, L. \& Forzza, R.C. 2002. Orquídeas do Parque Estadual de Ibitipoca. Boletim da Coordenadoria das Associações Orquidófilas do Brasil 48: 35-40.

Menini Neto, L.; Almeida, V.R. \& Forzza, R.C. 2004. A família Orchidaceae na Reserva Biológica da Represa do Grama - Descoberto, Minas Gerais, Brasil. Rodriguésia 55: 137-156.

Miller, D.; Warren, R. \& Miller, I.M. 1996. Orquídeas do alto da serra da mata atlântica pluvial do sudeste do Brasil. Rio de Janeiro, Lis Gráfica e Editora.

Munhoz, C.B.R. \& Proença, C.E.B. 1998. Composição florística do município de Alto Paraíso de Goiás na Chapada dos Veadeiros. Boletim do Herbário Ezechias Paulo Heringer 3: 102-150.

Pabst, J.F.G. \& Dungs, F. 1975. Orchidaceae Brasilienses. v. I. Hildesheim, Kurt Schmersow.

Pabst, J.F.G. \& Dungs, F. 1977. Orchidaceae Brasilienses. v. II. Hildesheim, Kurt Schmersow.

Pedralli, G.; Freitas, V.L.O.; Meyer, S.T.; Teixeira, M.C.B. \& Gonçalves, A.P.S. 1997. Levantamento florístico na estação ecológica do Tripuí, Ouro Preto, MG. Acta Botanica Brasilica 11: 191-213.

Peron, M.V. 1989. Listagem preliminar da flora fanerogâmica dos campos rupestres do Parque Estadual do Itacolomi - Ouro Preto/Mariana, MG. Rodriguésia 67: 63-69.

Pirani, J.R.; Giulietti, A.M.; Mello-Silva, R. \& Meguro, M. 1994. Checklist and patterns of geographic distribution of the vegetation of Serra do Ambrósio, Minas Gerais, Brazil. Revista Brasileira de Botânica 17: 133-147.

Pirani, J.R.; Mello-Silva, R. \& Giulietti, A.M. 2003. Flora de Grão Mogol, Minas Gerais, Brasil. Boletim de Botânica da Universidade de São Paulo 21: 1-24.

Ribeiro, J.E.L.S.; Hopkins, M.J.G.; Vicentini, A.; Sothers, C.A.; Costa, M.A.S.; Brito, J.M.; Souza, M.A.D.; Martins, L.H.P.; Lohmann, L.G.; Assunção, P.A.C.L.; Pereira, E.C.; Silva, C.F.; Mesquita, M.R. \& Procópio, L.C. 1999. Flora da Reserva Ducke: Guia de identificação das plantas vasculares de uma floresta de terra firme na Amazônia Central. Manaus, Instituto Nacional de Pesquisas da Amazônia.
Rodela, L.C. 1998. Cerrados de altitude e campos rupestres do Parque Estadual de Ibitipoca, sudeste de Minas Gerais: Distribuição e florística por subfisionomias da vegetação. Revista do Departamento de Geografia 12: 163-189.

Rodrigues, J.B. 1877. Genera et Species Orchidearum Novarum. v. 1, Rio de Janeiro, Typographia Nacional.

Rodrigues, J.B. 1882. Genera et Species Orchidearum Novarum. v. 2. Rio de Janeiro, Typographia Nacional.

Saint-Hilaire, A.F.C. 1822. Segunda viagem do Rio de Janeiro a Minas Gerais e São Paulo. (trad. Vivaldi Moreira, 1974). Belo Horizonte, Ed. Itatiaia.

Salimena-Pires, F.R. 1997. Aspectos fisionômicos e vegetacionais do Parque Estadual de Ibitipoca, Minas Gerais, Brasil. Pp. 61-60. In: G.C. Rocha (coord.). Anais do $1^{\circ}$ Seminário de Pesquisa sobre o Parque Estadual de Ibitipoca. Juiz de Fora, Núcleo de Pesquisa em Zoneamento Ambiental da UFJF.

Silveira, A.A. 1928. Floralia Montium. v. 2. Belo Horizonte, Imprensa Official.

Silveira, E.C.; Cardoso, A.L.R.; Ilkiu-Borges, A.L. \& Atzingen, N.V. 1995. Flora orquidológica da Serra dos Carajás, estado do Pará. Boletim do Museu Paraense Emílio Goeldi, Série Botânica 11: 75-87.

Sprunger, S. (ed.); Cribb, P. \& Toscano-de-Brito, A.L.V. (colabs.). 1996. João Barbosa Rodrigues - Iconographie des orchidées du Brésil. v. 1, The illustrations. Basle, Friedrich Reinhardt Verlag.

Stannard, B.L. (ed.). 1995. Flora of the Pico das Almas Chapada Diamantina, Bahia, Brazil. Kew, Royal Botanic Gardens.

Toscano-de-Brito, A.L.V. 1995. Orchidaceae. Pp. 725-767. In: B.L. Stannard (ed.). Flora of the Pico das Almas - Chapada Diamantina, Bahia, Brazil. Kew, Royal Botanic Gardens.

Urban, I. 1906. Vitae Itineraque Collectorum Botanicorum, Notae Collaboratorum Biographicae, Florae Brasiliensis Ratio Edendi Chronologica, Systema, Index Familiarum. Pp. 1-487. In: C.F.P. Martius; A.G. Eichler \& I. Urban (eds.). Flora brasiliensis 1(1). Monachii, Typographia Regia.

Vitta, F. 2002. Diversidade e conservação da flora nos campos rupestres da Cadeia do Espinhaço em Minas Gerais. Pp. 90-94. In: E.L. Araújo; A.N. Moura; E.V.S.B. Sampaio; L.M.S. Gestinari \& J.M.T. Carneiro (eds.). Biodiversidade, Conservação e Uso Sustentável da Flora do Brasil. Recife, Universidade Federal Rural de Pernambuco/Sociedade Botânica do Brasil.

Toscano-de-Brito, A.L.V. \& Cribb, P. 2005. Orquídeas da Chapada Diamantina. São Paulo, Nova Fronteira.

World Checklist of Monocots. 2004. The Board of Trustees of the Royal Botanic Gardens, Kew. Published on the Internet; http://www.kew.org/monocotChecklist/ (Acesso em: 26/05/2005).

Zappi, D.C.; Lucas, E.; Stannard, B.L.; Lughadha, E.N.; Pirani, J.R.; Queiroz, L.P.; Atkins, S.; Hind, D.J.N.; Giulietti, A.M.; Harley, R.M. \& Carvalho, A.M. 2003. Lista das plantas vasculares de Catolés, Chapada Diamantina, Bahia, Brasil. Boletim de Botânica da Universidade de São Paulo 21: 345-389. 